\title{
An Adult Plant Stripe Rust Resistance Gene Maps on Chromosome 7A of Australian Wheat Cultivar Axe
}

\author{
Mehwish Kanwal \\ The University of Sydney \\ Naeela Qureshi \\ DPI: Victoria Department of Jobs Precincts and Regions \\ Mesfin Gessese \\ The University of Sydney \\ Kerrie Forrest \\ DPI: Victoria Department of Jobs Precincts and Regions \\ Prashanth Babu \\ The University of Sydney \\ Harbans Bariana \\ The University of Sydney \\ Urmil Bansal ( $\square$ urmil.bansal@sydney.edu.au ) \\ University of Sydney https://orcid.org/0000-0003-1119-4464
}

\section{Original Article}

Keywords: Adult plant resistance, stripe rust, targeted genotype-by-sequencing, wheat

Posted Date: February 11th, 2021

DOI: https://doi.org/10.21203/rs.3.rs-183642/v1

License: (c) (i) This work is licensed under a Creative Commons Attribution 4.0 International License. Read Full License

Version of Record: A version of this preprint was published at Theoretical and Applied Genetics on April 10th, 2021. See the published version at https://doi.org/10.1007/s00122-021-03818-x. 


\section{Abstract}

Australian wheat cultivar Axe produced resistant to moderately resistant stripe rust responses under field conditions and was exhibiting seedling response varying from $33 \mathrm{C}$ to $3+$ under greenhouse conditions. Experiments covering tests at different growth stages $\left(2^{\text {nd }}, 3^{\text {rd }}\right.$ and $4^{\text {th }}$ leaf stages) demonstrated the clear expression of resistance at the $4^{\text {th }}$ leaf stage under controlled-environment greenhouse conditions. A recombinant inbred line (RIL) population was developed from the Axe/Nyabing-3 (Nyb) cross. Genetic analysis of Axe/Nyb RIL population in the greenhouse at the $4^{\text {th }}$ leaf stage showed monogenic inheritance of stripe rust resistance. Selective genotyping using the iSelect 90K Infinium SNP genotyping array was performed and the resistance locus was mapped to long arm of chromosome 7A and named Yr75. The Axe/Nyb RIL population was genotyped using a targeted genotype-by-sequencing (tGBS) assay and the resistance-linked SNPs were converted into kompetitive allele specific PCR (KASP) markers. These markers were tested on the entire Axe/Nyb RIL population and markers sunKASP_430 and sunKASP_427 showed close association with $Y r 75$ in Axe/Nyabing-3 RIL population. A high-resolution mapping family of $1032 \mathrm{~F}_{2}$ plants from the Axe/Nyb cross was developed and genotyped with sunKASP_430 and sunKASP_427 and these markers flanked $Y r 75$ at 0.3 $\mathrm{CM}$ and $0.4 \mathrm{cM}$, respectively. These markers covers $1.24 \mathrm{Mb}$ of the physical map of Chinese Spring and can be used for future map-based cloning of this gene.

\section{Introduction}

Stripe rust or yellow rust, caused by Puccinia striiformis f. sp. tritici (Pst), ranks high among fungal diseases of wheat worldwide. This disease has the potential to cause almost 1 billion AUD of losses in Australia (Murray and Brennan 2009). Previously, this disease was endemic to cooler wheat-growing regions, but in the last two decades it has adapted to relatively warmer regions causing worldwide expansion and leading to many destructive pandemics (Ali et al. 2014).

The global progression and rapid evolution of stripe rust has led to increased application of fungicides, effectively decreasing the economic losses in several epidemics (Carmona et al. 2020), but the use of fungicides is neither environment-friendly nor cost-effective control measure. Experiments have also been conducted on the biological control of stripe rust, but no significant reduction in severity of disease has been noted. This approach is also not practically viable (Reiss and Jørgensen 2017; Feodorova-Fedotova et al. 2019). Both these control measures are resource-demanding and unprofitable for farmers with small land holdings in developing nations. In contrast, breeding for rust resistance is considered a preferred method to effectively control this disease due to its eco-friendly and economic nature (Bariana et al. 2007a).

Resistance to stripe rust has been classified into two broad classes (Bariana 2003; Chen 2005) often referred to as overall resistance (also known as all stage resistance; ASR) and adult plant resistance (APR). Genes that condition ASR follow gene-for-gene model (Flor 1942) and exhibit high levels of resistance. Deployment of ASR genes individually in wheat cultivars makes them vulnerable to breakdown through acquisition of virulence in pathogen populations (Bariana et al. 2016). The APR genes express resistance at post-seedling stages and show non-hypersensitive reactions and slow disease development on genotypes carrying these genes. This type of resistance has also been referred to as partial or slow rusting resistance and is considered more durable (Caldwell 1968). Some APR genes conferred pleiotropic resistance towards multiple diseases including stripe rust, leaf rust, stem rust and powdery mildew. These include Yr18/Lr34/Sr57/Pm38; Yr29/Lr46/Sr58/Pm39 and Yr46/Lr67/Sr55/Pm46 (https://wheat.pw.usda.gov/GG3/wgc). A third category of resistance that expresses clearly at the 3rd to 4 th leaf stages, but behaves like ASR in expression and can be referred to as mid-stage resistance (MSR) (Chhetri et al. 2016a), for example; Yr58 (Chhetri et al. 2016b), Lr48 (Nsabiyera et al. 2016) and Lr49 (Nsabiyera et al. 2020).

To date, 83 stripe rust resistance genes have been formally designated (https://wheat.pw.usda.gov/GG3/wgc) and most of these genes belong to the ASR category. The availability of various high-throughput genotyping platforms (e.g. DArT array, DArTseq, 40K and 90K SNP arrays, genotyping-by-sequencing) have accelerated the discovery of new disease resistance loci and the development of trait-linked molecular markers. Moreover, complete genome assembly of common wheat genotype Chinese Spring IWGSC RefSeq v1.0 (IWGSC, 2018) and IWGSC RefSeq v2.0

(https://urgi.versailles.inra.fr/download/iwgsc/IWGSC_RefSeq_Assemblies/v2.0/) have offered opportunities for fine mapping and map-based cloning of loci that control economic tratis.

Stripe rust resistance genes Yr26 (Wu et al. 2018), Yr29 (Cobo et al. 2019) and Yr47 (Qureshi et al. 2017) have recently been fine mapped. Resistance genes Yr5, Yr7, YrSp (Marchal et al. 2018), Yr15 (Klymiuk et al. 2018), Yr18/Lr34 (Krattinger et al. 2009), Yr36 (Fu et al. 2009) and Yr46 (Moore et al. 2015) have been cloned using these new tools. Axe, an Australian cultivar released in 2015 by Australian Grain Technologies, was susceptible at the seedling stage against Australian Pst pathotypes and showed high level of resistance at the adult plant stage. A recombinant inbred line (RIL) population was developed for genetic analysis of stripe rust resistance in cultivar Axe and molecular mapping of gene (s) involved in expression of resistance.

\section{Materials And Methods Population development}

Cultivar Axe was crossed with a susceptible genotype Nybing-3, a selection from cultivar Nyabing. $F_{1}$ seeds were grown and harvested separately for producing the $F_{2}$ population. Individual $F_{2}$ seeds were planted $10 \mathrm{~cm}$ apart in the field and each plant was harvested separately. A single spike was harvested from each greenhouse grown $\mathrm{F}_{3}$ family to generate an $\mathrm{F}_{4}$ population. Similarly, single head harvest from $\mathrm{F}_{4}$ generation led to the production of an $\mathrm{F}_{5}$ population. Single seed from each $F_{5}$ line was grown and the whole plant was harvested to raise an $F_{6}$ generation. The final set of 151 lines is referred to as the Axe/Nyb recombinant inbred line (RIL) population.

\section{Greenhouse screening}


The Axe/Nyb RIL population was screened in the greenhouse against the most prevalent Pst pathotype 134E16A+Yr17+Yr27+ (Plant Breeding Institute culture no. 617). Eight to 10 seeds from each RIL (four RILs per pot) were sown in 9-cm diameter pots filled with a potting mixture comprising of composted pine bark and sand in a 2:1 ratio, followed by fertilizer treatment (25g Aquasol®; Hortico Pty. Ltd., Revesby, NSW, Australia /10L of water for 100 pots). Seedlings were fertilised weekly with Urea at the same rate as Aquasol. Inoculations were carried out at the $4^{\text {th }}$ leaf stage in a specialized inoculation chamber by atomising urediniospores of Pst pathotype 134E16 A+Yr17+Yr27+ suspended in light mineral oil (Isopar L, 5 mg spores $10 \mathrm{ml}^{-1}$ of oil as solvent) using a hydrocarbon propellant pressure pack. After inoculation, plants were moved to luke-warm water-filled steel trays covered with polythene hoods in the incubation room set at $9-12{ }^{\circ} \mathrm{C}$. Following 24 hours of incubation, plants were transferred to microclimate rooms (automated temperature and irrigation control) set at $17^{\circ} \mathrm{C}$. Rust response assessments were performed 16 -18 days after inoculation using a 0 to 4 scale described in Mclntosh et al. (1995.) The Axe/Nyb RILs were classified as homozygous resistant (HR), homozygous susceptible (HS) and segregating (this class can have very low frequency and sometime could represent a mixture).

\section{Molecular mapping}

\section{DNA extraction}

DNA from 10-12 days old seedings of each Axe/Nyb RIL and parents was extracted and quantified following a modified CTAB method outlined in Bansal et al. (2014).

\section{Chromosomal location of stripe rust resistance}

\section{Selective genotyping}

For identification of chromosomal location of the resistance locus, selective genotyping was carried out on eight resistant and eight susceptible RILs using an Illumina iSelect 90K Infinium SNP genotyping array (Wang et al. 2014). The stripe rust response-linked SNPs were converted into kompetitive allele-specific polymerase chain reaction (KASP) assays and were tested on parental lines (Axe and Nyb) using the protocol described by LGC (Laboratory of the Government Chemist) genomics, UK (www.biosearchtech.com/ngs). The KASP markers which gave clear clusters were genotyped on the entire RIL population for construction of a linkage map.

\section{KASP genotyping}

All KASP assays in this study were genotyped following the procedure described in Pakeerathan et al. (2019).

\section{Detailed mapping using a targeted genotyping-by-sequencing (tGBS) assay}

Once the genomic region that controls stripe rust resistance was identified through selective genotyping, we used targeted genotyping-by-sequencing (tGBS) assay for saturation of the map. This service was provided by Agriculture Victoria, Agribio, Bundoora, Victoria. The purpose of the tGBS assay was to capture additional polymorphisms between parents by sequencing the genomic region of interest. The genetic map was constructed using the R package ASMap (Taylor and Butler 2017) and imputations for missing SNP data were performed using Impute v2.2 (Sargolzaei et al. 2014).

\section{Marker design}

An automated bioinformatic pipeline PolyMarker (Ramirez-Gonzalez et al. 2015) was used for designing KASP markers from the SNPs associated with stripe rust resistance identified through tGBS mapping. Allele specific primers A1 and A2 were tagged with diagnostic sequences for fluorescent dyes FAM (gaaggtgaccaagttcatgct) and HEX (gaaggtcggagtcaacggatt) at their 5囚, respectively.

\section{High-resolution mapping}

A set of seven KASP markers from the tGBS assay were genotyped on the entire Axe/Nyb RIL population (154 lines) and these markers were incorporated into the initial map. Following this, a large mapping population of $1032 \mathrm{~F}_{2}$ plants (2064 gametes) from an Axe/Nyb cross was developed. DNA from this population was extracted using a modified SDS (sodium dodecyl sulfate) DNA extraction protocol (M. Pourkheirandish personal communication). Leaf tissue

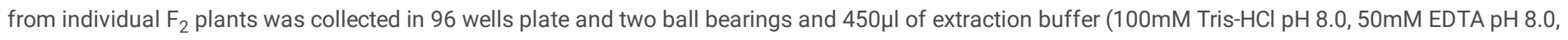

$500 \mathrm{mM} \mathrm{NaCl}$ and $10 \mathrm{mM} 2$-mercaptoethanol) was added in each well and samples were crushed in a tissue lyser (1600 MiniG ${ }^{\mathrm{TM}}$ ) for 3 minutes. After crushing, $60 \mu \mathrm{l}$ of $10 \%$ SDS buffer was added and samples were inverted vigorously for homogenous mixing. Samples were then incubated at $65{ }^{\circ} \mathrm{C}$ for 60 min and the plate was centrifuged for $1 \mathrm{~min}$ at $3600 \mathrm{rpm}$. After centrifugation, $200 \mu \mathrm{l}$ of $7.5 \mathrm{M}$ ammonium acetate was added and the plate was shaken vigorously, followed by incubation for $60 \mathrm{~min}$ at $4{ }^{\circ} \mathrm{C}$. After centrifugation at $1000 \mathrm{rpm}$ for $1 \mathrm{~min}, 300 \mu \mathrm{l}$ of chloroform was added and the plate was again shaken vigorously, followed by centrifugation for $10 \mathrm{~min}$ at $4800 \mathrm{rpm}$. One hundred $\mu$ l of supernatant was added to100 $\mu$ l of chilled isopropanol in new plate and 
mixed gently. After centrifugation for $10 \mathrm{~min}$ at $4800 \mathrm{rpm}$, the isopropanol was removed and the pellet was resuspended in $100 \mu \mathrm{l}$ of $1 \mathrm{M}$ Tris $\mathrm{HCl}(\mathrm{pH} 8)$ containing $10 \mathrm{mM}$ of RNase, with incubation at $37^{\circ} \mathrm{C}$ for $1-2$ hours.

Yr75 Flanking markers were tested on a large $F_{2}$ population. Recombinants were phenotyped in the $F_{3}$ generation against Pst pathotype $134 E 16 A+17+27$ to refine the location of the stripe rust resistance locus.

\section{Statistical analysis and genetic mapping}

Chi-squared $\left(\chi^{2}\right)$ analysis was used to identify segregation distortion among markers located near the resistance locus. The KASP and SSR marker data were converted to ' $A$ ' for Axe allele and 'B' for Nyb allele and ' $H$ ' for heterozygotes for mapping. Genetic linkage maps were constructed using MapManager QTXb20 (Manly et al. 2001) with the Kosambi map function (Kosambi 1943) and then presented graphically using MapChart version 2.3 (Voorrips 2002 ). The physical position of markers that flanked the resistance locus and structural variation in the genomic region was investigated using the tool Pretzel (Keeble-Gagnère et al. 2019) which compares genetic and physical maps.

\section{Results}

\section{Greenhouse screening}

The Axe/Nyb RIL population was inoculated with the Pst pathotype $134 \mathrm{E} 16 \mathrm{~A}+\mathrm{Yr} 17+\mathrm{Yr} 27+$ under greenhouse conditions at the $2^{\text {nd }}, 3^{\text {rd, }}$ and $4^{\text {th }}$ leaf stages. $^{\text {th }}$ Expression of resistance was not clear at the $2^{\text {nd }}$ to $3^{\text {rd }}$ leaf stages; however, a clear expression of resistance was observed at the $4^{\text {th }}$ leaf stage. The resistant parent Axe produced infection type (IT) ; $1^{\top} \mathrm{C}$ and the susceptible parent produced IT3+ (Figure 5.1). Infection types among resistant RILs ranged from IT1C to IT23C. These results suggested that resistance carried by Axe does not typically belong to the either of the currently defined ASR or APR categories.

The Axe/Nyb RILs were classified as homozygous resistant (HR=1C to 23C) and homozygous susceptible (HS=3+). Eighty-four lines were placed in the HR class, while 67 were categorised as HS. Chi-squared analysis of stripe rust response variation among RILs conformed to segregation at a single locus (Table 5.1). The underlying stripe rust resistance locus was named YrAxe.

Table 1

Frequency distribution of Axe/Nyb RILs when tested against Pst pathotype 134E16A+Yr17+Yr27+

\begin{tabular}{|c|c|c|c|}
\hline \multirow[t]{2}{*}{ Phenotype } & \multicolumn{2}{|c|}{ Number of RILs } & \multirow[t]{2}{*}{$\chi^{2}(1: 1)$} \\
\hline & Observed & Expected & \\
\hline Homozygous resistant & 84 & 75.5 & 0.96 \\
\hline Homozygous susceptible & 67 & 75.5 & 0.96 \\
\hline Total & 151 & 151 & 1.92 \\
\hline
\end{tabular}

\section{Molecular mapping of YrAxe}

Selective genotyping with the 90K SNP array was used to identify the chromosomal location of YrAxe. Of 306 linked SNPs, 140 SNPs were mapped in chromosome 7AL, 55 in 7BL and seven on 7DL of the wheat consensus map (Wang et al. 2014). Among the 7AL SNPs, 128 SNPs spanned a region of $708,363,089$ bp to $726,684,629$ bp of the Chinese Spring physical map (IWGSC RefSeq v1.0) and showed strong linkage with $Y r A x e$. One SNP from each representative LD block (28 SNPs in total) was converted into a KASP assay. Two KASP markers KASP_34640, and KASP_38710, clearly differentiated the parents (Table 5.2) and KASP_39562 produced heterozygous allele for Nyb.

Table 2

List of YrAxelinked KASP markers developed from the iSelect 90K SNP genotyping

\begin{tabular}{|c|c|c|c|c|c|}
\hline Marker & SNP & Position in $90 \mathrm{~K}$ map $(\mathrm{cM})^{*}$ & \multicolumn{3}{|l|}{ Primer sequence } \\
\hline KASP_34640 & {$[A / G]$} & 216.36 & Aagataaaatcgtccatcaagtct & aagataaaatcgtccatcaagtcc & atgaaggacgacgcagacac \\
\hline KASP_38710 & {$[\mathrm{T} / \mathrm{C}]$} & 212.97 & Ggagatgacaggtcaaaactatcat & ggagatgacaggtcaaaactatcac & tatgcgtaggccttcccgta \\
\hline KASP_39562 & {$[\mathrm{T} / \mathrm{C}]$} & Not known & Cacggataggctcttgcttt & cacggataggctcttgcttc & tgggaagcttatgcctggt \\
\hline
\end{tabular}

Page $4 / 10$ 
YrAxe was mapped on the long arm of chromosome 7A. The SSR marker, cfa2040, previously mapped on chromosome 7A was used to confirm the map location of linked SNP markers and was genotyped on the entire Axe/Nyb RIL population. YrAxe was flanked by $c f a 2040$ and KASP_34640. Since no other stripe rust resistance gene was previously located in the region flanked by $c f a 2040$ and KASP_34640, YrAxe was permanently named Yr75.

\section{Detailed mapping of Yr75}

\section{Low-resolution mapping}

Seven SNPs from the tGBS assay that showed close association with $Y r 75$ were converted into KASP markers (sunKASP_425, sunKASP_426, sunKASP_427, sunKASP_428, sunKASP_429, sunKASP_430, sunKASP_431). Primer sequences of these markers are given in Table 3. These markers differentiated parents clearly and were genotyped on the entire Axe/Nyb RIL population. A genetic map carrying $Y r 75$ was developed including two $90 \mathrm{~K}$ SNPs and $c f a 2040$. Marker sunKASP_427 mapped at $0.7 \mathrm{cM}$ proximally to $\mathrm{Yr} 75$ while sunKASP_430 co-segregated with the gene (Figure 2a). The physical positions of flanking markers in Chinese Spring (IWGSC RefSeq v1.0) were at 719,076,651 (sunKASP_427) and 717,832,538 bp (sunKASP_430) (Figure 3).

Table 3

List of Yr75tinked KASP markers developed from the tGBS assay

\begin{tabular}{|c|c|c|c|c|c|c|c|}
\hline \multirow[t]{2}{*}{ Marker } & \multirow[t]{2}{*}{ tGBS marker name } & \multirow[t]{2}{*}{ SNP } & \multirow{2}{*}{$\begin{array}{l}\text { Super } \\
\text { scaffold }\end{array}$} & \multirow{2}{*}{$\begin{array}{l}\text { Position in } \\
\text { IWGSC v1.0 } \\
\text { wheat } \\
\text { genome } \\
\text { (bp) }\end{array}$} & \multicolumn{3}{|l|}{ Primer sequence } \\
\hline & & & & & Allele 1 & Allele 2 & Common \\
\hline sunKASP_425 & $\begin{array}{l}\text { scaffold134444|TaGBSv2- } \\
\text { 5557_274769 }\end{array}$ & {$[\mathrm{T} / \mathrm{C}]$} & 31 & $714,587,271$ & tcatggtcaagttcaacggt & tcatggtcaagttcaacggc & tcgctaggggagtgc \\
\hline sunKASP_426 & $\begin{array}{l}\text { scaffold43271- } \\
\text { 1|TaGBSv2-5558_88393 }\end{array}$ & {$[\mathrm{C} / \mathrm{G}]$} & 32 & $715,122,740$ & ctattgccctcctcatcgatg & ctattgccctcctcatcgatc & gagggaggaagcg، \\
\hline sunKASP_427 & $\begin{array}{l}\text { scaffold96044|TaGBSv2- } \\
\text { 11657_1099665 }\end{array}$ & {$[A / G]$} & 32 & $719,076,651$ & cgacgacgtccaaagactaat & cgacgacgtccaaagactaac & gcaacaacacacta! \\
\hline sunKASP_428 & $\begin{array}{l}\text { scaffold43271- } \\
\text { 1|TaGBSv2- } \\
\text { 5562_2434232 }\end{array}$ & {$[\mathrm{T} / \mathrm{A}]$} & 32 & $717,468,579$ & cacagccgcggaaagact & cacagccgcggaaagaca & tgcggttgccttgttat \\
\hline sunKASP_429 & $\begin{array}{l}\text { scaffold43271- } \\
\text { 1|TaGBSv2- } \\
\text { 11654_2223053 }\end{array}$ & {$[\mathrm{T} / \mathrm{C}]$} & 32 & $717,257,400$ & agccgctcgaactacattttt & agccgctcgaactacattttc & gctgtgcacagtatg: \\
\hline sunKASP_430 & $\begin{array}{l}\text { scaffold43271- } \\
\text { 1|TaGBSv2- } \\
\text { 11655_2798191 }\end{array}$ & {$[A / G]$} & 32 & $717,832,538$ & agcgcagagagatgcagt & agcgcagagagatgcagc & agacctgcggtagct \\
\hline sunKASP_431 & $\begin{array}{l}\text { scaffold96044|TaGBSv2- } \\
\text { 5564_877551 }\end{array}$ & {$[\mathrm{C} / \mathrm{A}]$} & 34 & $718,854,537$ & tggtccgccatgaagtcc & tggtccgccatgaagtca & acgaccttctgcacg1 \\
\hline
\end{tabular}

\section{High-resolution mapping}

Markers sunKASP_425, sunKASP_426, sunKASP_427, sunKASP_428, sunKASP_429, sunKASP_430 and sunKASP_431 from the Axe/Nyb low resolution map were genotyped on 1032 individual $\mathrm{F}_{2}$ plants (2064 gametes) to refine recombination in the region. KASP_34640 and KASP_38710 did not produce clear clusters for heterozygotes therefore were not included in mapping. Marker cfa2040 is a multiallelic locus (amplify alleles on chromosome 7A, 7B and 7D), size difference was only $2 \mathrm{bp}(7 \mathrm{~A})$ which can be assessed clearly in the RIL population, but it is very difficult to score in $\mathrm{F}_{2}$ population, therefore this marker was also not used for genotyping. Thirty recombinants were identified and grown for progeny testing. These progenies were scored at the $4^{\text {th }}$ leaf stage and a highresolution map was constructed. Markers sunKASP_427 and sunKAS_430 flanked Yr75 at $0.4 \mathrm{cM}$, and $0.3 \mathrm{cM}$ proximally and distally, respectively (Figure 2b).

\section{Discussion}

This study demonstrated the expression of stripe rust resistance at the 4th leaf stage in wheat cultivar Axe and the underlying locus was mapped on the long arm of chromosome 7A. As none of the previously known stripe rust resistance genes mapped in this region, the resistance locus was permanently named Yr75. The expression of stripe rust resistance at post seedling stages was previously described by Chhetri et al. 2016 in a landrace W195 from India and in an Australian cultivar Sentinel by Chemayek (2016). This class of genes should be treated as a different category and ideally fit into the MSR category and unlike typical APR genes Yr18 and Yr29 (Lagudah et al. 2006; Mclntosh 1992; Singh 1992).

This investigation used the state-of-the-art technologies and sequence information for detailed mapping of the chromosome 7A region that carried $Y r 75$. Several studies have used an integrated approach for development of molecular markers for rust resistance which include $L r 49$ (Nsabiyera et al. 2020 ), $Y r 47$ (Qureshi et al. 2017) and Yr26 (Wu et al. 2018). 
The Axe/Nyb map was saturated through a targeted genotyping-by-sequencing (tGBS) assay that generates sequence data for genomic regions surrounding known exome-derived SNPs. SNPs identified from the tGBS analysis, 90K Infinium assay and SSR marker cfa2040 led to the development of a low-resolution map with $0.7 \mathrm{cM}$ interval with sunKASP_427 and Yr75-sunKASP_430. Markers cfa2040 and KASP_34640 were located in the tGBS scaffolds scaffold43271-1 and scaffold96044, respectively, from which Yr75-flanking markers were devloped. Due to the lack of good clustering and similar positioning in the scafoolds these markers were not genotyped in the high resolution population. Markers devloped from tGBS SNPs were genotyped on a high-resolution Axe/Nyb $\mathrm{F}_{2}$ population and genetic distances of $0.4 \mathrm{cM}$ for sunKASP_427 (11 recombinants) and $0.3 \mathrm{cM}$ for sunKASP_430 (10 recombinants) from Yr75 were obsereved. These markers were devloped from two scaffolds (scaffold96044 and scaffold43271-1) and were represented in the superscaffold 32 of the IWGSC 1.0 genome assembly (IWGSC. 2018). The tGBS approach has been used for mapping and development of molecular markers for only a few genes including Yr82 (Pakeerathan et al. 2019) Sr26 (Qureshi et al. 2018) and Yr26 (Wu et al. 2018) in wheat.

Despite the low genetic distance (1.24 Mb physical disctance; IWGSC RefSeq v1.0 genome assembly) between closely linked markers sunKASP_427 and sunKASP_430 and $Y r 75$, we were unable to validate these markers on a diverse set of wheat genotypes, which suggested either chromosomal rearrangement or the supressed recombination in this region. $Y r 75$ is located near the telomere of the 7A chromosome, where frequent recombination should be expected. The existence of suppressed recombination is also supported by previous studies on pleiotropic locus Sr15/Lr20/Pm1 (Sears and Briggle 1969; Watson and Luig 1966) on chromosome 7AL, which showed a total genetic distance of $9.6 \mathrm{cM}$ flanked by markers psr148 and psr687 (Gale 1995). The fine mapping of chromosome 7AL later revealed that the markers cdo347, psr121, and ksuh9 covered a region of $30 \mathrm{cM}$ (Peña et al. 1997). Genetic mapping of the Lr20-Pm1 in three different $F_{2}$ populations also revealed that markers at the distal end were completely linked with $L$ r20-Pm 1 locus, but at the proximal end there was a discrepancy compared to previous map for this locus (Neu et al. 2002). These results also suggested reduced recombination in this region and/or chromosomal rearrangements. Suppressed recombination was also reported in another study for root lesion nematode gene R/nn1 which is tightly linked with Sr15/ Lr20 locus (Jayatilake et al. 2013). These results supported the hypothesis that terminal region of chromosome 7A is one of the complex region resulted due to several chromosomal abberations (Badaeva et al. 2007). To move forward, different appraoches such as flow sorting of chromosome of the line carrying the target locus could be helpful in saturating the region and finding closer markers. Further study on this region by using advanced genomic techniques including sequence capture and CRIPSR mediated genetic manipulation will facilitate the unexplained reason for complexity of this region.

In conclusion, this study provided a new adult plant stripe rust resistance gene $Y r 75$ and tightly linked markers for marker-assisted selection for this gene in breeding material following parental polymorphism check. These results will be useful in map based cloning of Yr75.

\section{Declarations \\ Funding:}

Grain Research Development Corporation, Australia.

\section{Conflicts of interest/Competing interests:}

The authors declare that they have no conflict of interest.

\section{Availability of data and material:}

All data are given in the manuscript

\section{Code availability:}

Publicly available software's are used in this study

\section{Authors' contributions:}

UB \& HB planned the study, MK drafted the manuscript; MK \& MG developed segregating population and performed phenotyping, KF and NQ performed genotyping and tGBS analysis, UB and MK designed the KASP primers and MK and PB performed KASP genotyping, UB, HB, KF, and NQ edited the manuscript.

\section{Keymessage:}

An adult plant stripe rust resistance gene Yr75 was fine mapped on chromosome 7AL which is a step closure for future map-based cloning for this gene.

\section{References}

1. Ali S, Gladieux P, Leconte M, Gautier A, Justesen AF, Hovmøller MS, Enjalbert J, De Vallavieille-Pope C (2014) Origin, migration routes and worldwide population genetic structure of the wheat yellow rust pathogen Puccinia striiformis $\mathrm{f}$. sp. tritici. PLoS pathogens 10:1-12 
2. Badaeva ED, DedKova OS, Gay G, Pukhalskyi VA, Zelenin AV, Bernard S, Bernard M (2007) Chromosomal rearrangements in wheat: their types and distribution. Genome 50:907-926

3. Bansal U, Kazi AG, Singh B, Hare RA, Bariana H (2014) Mapping of durable stripe rust resistance in a durum wheat cultivar Wollaroi. Mol Breed 33:51-59

4. Bariana H (2003) Breeding for disease resistance. In Encyclopedia of applied plant sciences. In: Thomas B, Murphy DJ, Murray BG (eds) Academic Press Harcourt, 244-253

5. Bariana H, Brown G, Bansal U, Miah H, Standen G, Lu M (2007a) Breeding triple rust resistant wheat cultivars for Australia using conventional and markerassisted selection technologies. Austr J Agri Res 58:576-587

6. Bariana H, Forrest K, Qureshi N, Miah H, Hayden M, Bansal U (2016) Adult plant stripe rust resistance gene Yr71 maps close to Lr24 in chromosome 3D of common wheat. Mol Breed 36: 98

7. Caldwell RM (1968) Breeding for general and/or specific plant disease resistance. Proc $3^{\text {rd }}$ Int Wheat Genetics Sym, Australian Academy of Science, Canberra, Australia, pp 263-272

8. Carmona M, Sautua F, Pérez-Hérnandez O, Reis EM (2020) Role of fungicide applications. Front Plant Sci 11:733-755

9. Chemayek B (2016) Studies on resistance to biotic and abiotic stresses in wheat. PhD Thesis, The University of Sydney, Australia.

10. Chen X (2005) Epidemiology and control of stripe rust (Puccinia striiformis f. sp. tritici) on wheat. Canadian J Plant Pathol 27: $314-337$

11. Chhetri M, Bansal U, Toor A, Lagudah E, Bariana H (2016a) Genomic regions conferring resistance to rust diseases of wheat in a W195/BTSS mapping population. Euphytica 209:637-649

12. Chhetri M, Bariana H, Kandiah P, Bansal U (2016b) Yr58: a new stripe rust resistance gene and its interaction with Yr46 for enhanced resistance. Phytopathol 106:1530-1534

13. Cobo N, Wanjugi H, Lagudah E, Dubcovsky J (2019) A high-resolution map of wheat QYr. ucW-1BL, an adult plant stripe rust resistance locus in the same chromosomal region as Yr29. The plant genome, 12:1-15

14. Feodorova-Fedotova L, Bankina B ,Strazdina V (2019) Possibilities for the biological control of yellow rust (Puccinia striiformis $\mathrm{f}$. sp. tritici) in winter wheat in Latvia in 2017-2018. Agro Res 17: 716-724

15. Flor HH (1942) Inheritance of pathogenicity in a cross between physiologic races 22 and 24 of Melampsora lini. Phytopathol $32: 5$

16. Fu D, Uauy C, Distelfeld A, Blechl A, Epstein L, Chen X, Dubcovsky J (2009) A kinase-START gene confers temperature-dependent resistance to wheat stripe rust. Sci 323:1357-1360

17. Gale M (1995) Genetic maps of hexaploid wheat. Proc $8^{\text {th }}$ Intl Wheat Genet Symp, 1995, pp 29-40

18. Jayatilake DV, Tucker E, Bariana H, Kuchel H, Edwards J, McKay A, Mather DE (2013) Genetic mapping and marker development for resistance of wheat against the root lesion nematode Pratylenchus neglectus. BMC Plant Biology 13: 230

19. Keeble-Gagnère G, Isdale D, Suchecki Rt, Kruger A, Lomas K, Carroll D , Tibbits J (2019) Integrating past, present and future wheat research with Pretzel. BioRxiv 517953

20. Klymiuk V, Yaniv E, Huang L, Raats D, Fatiukha A, Chen S, Chang W (2018) Cloning of the wheat Yr15 resistance gene sheds light on the plant tandem kinase-pseudokinase family. Nature Commun 9: 1-12

21. Kosambi DD (1943) The eslimation of map distances from recombination values. Ann Eugen 12:172-175

22. Krattinger SG, Lagudah ES, Spielmeyer W, Singh RP, Huerta-Espino J, McFadden H, Bossolini E, Selter LL, Keller BJS (2009) A putative ABC transporter confers durable resistance to multiple fungal pathogens in wheat. Sci 323:1360-1363

23. Lagudah E, McFadden H, Singh RP, Huerta-Espino J, Bariana H, Spielmeyer W (2006) Molecular genetic characterization of the Lr34/Yr18 slow rusting resistance gene region in wheat. Theor Appl Genet 114: 21-30

24. Manly KF, Cudmore JR, Meer JM (2001) Map Manager QTX, cross-platform software for genetic mapping. Mammalian Genome 12: $930-932$

25. Marchal C, Zhang J, Zhang P, Fenwick P, Steuernagel B, Adamski NM, Lagudah E (2018) BED-domain-containing immune receptors confer diverse resistance spectra to yellow rust. Nature Plants 4: 662-668

26. Mclntosh RA (1992) Close genetic linkage of genes conferring adult-plant resistance to leaf rust and stripe rust in wheat. Plant Pathol 41:523-527

27. McIntosh RA, Welling CR, Park RF (1995) Wheat rusts: an atlas of resistance genes. CSIRO, Melbourne, Australia, 200 pp

28. Moore JW, Herrera-Foessel S, Lan C, Schnippenkoetter W, Ayliffe M, Huerta-Espino J, Lillemo M, Viccars L, Milne R, Periyannan SJNg (2015) A recently evolved hexose transporter variant confers resistance to multiple pathogens in wheat. Nature Genet 47:1494-1498

29. Murray GM, Brennan JP (2009) Estimating disease losses to the Australian wheat industry. Australas Plant Pathol 38:558-570

30. Neu C, Stein N, Keller B (2002) Genetic mapping of the Lr20/Pm1 resistance locus reveals suppressed recombination on chromosome arm 7AL in hexaploid wheat. Genome 45:737-744

31. Nsabiyera V, Baranwal D, Qureshi N, Kay P, Forrest K, Valárik M, Bansal UK (2020) Fine mapping of Lr49 using 90k SNP chip array and flow-sorted chromosome sequencing in wheat. Front Plant Sci 10:1787

32. Nsabiyera V, Qureshi N, Bariana HS, Wong D, Forrest KL, Hayden MJ, Bansal UK (2016) Molecular markers for adult plant leaf rust resistance gene Lr48 in wheat. Mol Breed 36: 65

33. Pakeerathan K, Bariana H, Qureshi N, Wong D, Hayden M, Bansal U (2019) Identification of a new source of stripe rust resistance Yr82 in wheat. Theor Appl Genet 132: 3169-3176

34. Peña RCDL, Murray TD , Jones SS (1997) Identification of an RFLP interval containing Pch2 on chromosome 7AL in wheat. Genome 40: $249-252$

Page $7 / 10$ 
35. Qureshi N, Bariana H, Forrest K, Hayden M, Keller B, Wicker T, Bansal U (2017) Fine mapping of the chromosome 5B region carrying closely linked rust resistance genes Yr47 and Lr52 in wheat. Theor Appl Genet 130: 495-504

36. Qureshi N, Kandiah P, Gessese MK, Nsabiyera V, Wells V, Babu P, Wong D, Hayden M, Bariana H, Bansal U (2018). Development of co-dominant KASP markers co-segregating with Ug99 effective stem rust resistance gene Sr26 in wheat. Mol Breed 38:97

37. Ramirez-Gonzalez RH, Uauy C, Caccamo M (2015) PolyMarker: a fast polyploid primer design pipeline. Bioinformatics 31: $2038-2039$

38. Reiss A, Jørgensen LN (2017) Biological control of yellow rust of wheat (Puccinia striiformis) with Serenade® ASO (Bacillus subtilis strain QST713). Crop Prot 93: 1-8

39. Sargolzaei M, Chesnais JP, Schenkel FS (2014) A new approach for efficient genotype imputation using information from relatives. BMC Genomics 15: 478.

40. Sears ER, Briggle LW (1969) Mapping the gene Pm/ for resistance to Erysiphe graminis f. sp. tritici on chromosome 7A of wheat. Crop Sci 9: $96-97$

41. Singh RP (1992) Genetic association of leaf rust resistance gene Lr34 with adult plant resistance to stripe rust in bread wheat. Phytopathol (USA)

42. Taylor J, Butler D (2017) R package ASMap: efficient genetic linkage map construction and diagnosis. J Statistical Software 79:2869 DOI: 10.18637/jss.v079.i06

43. Voorrips RE (2002) MapChart: software for the graphical presentation of linkage maps and QTLs. J Hered 93: 77-78

44. Wang S, Wong D, Forrest K, Allen A, Chao S, Huang BE, Maccaferri M, Salvi S, Milner SG, Cattivelli L, Mastrangelo AM, Whan A, Stephen S, Barker G, Wieseke R, Plieske J, International Wheat Genome Sequencing C, Lillemo M, Mather D, Appels R, Dolferus R, Brown-Guedira G, Korol A, Akhunova AR, Feuillet C, Salse J, Morgante M, Pozniak C, Luo MC, Dvorak J, Morell M, Dubcovsky J, Ganal M, Tuberosa R, Lawley C, Mikoulitch I, Cavanagh C, Edwards KJ, Hayden M, Akhunov E (2014) Characterization of polyploid wheat genomic diversity using a high-density 90,000 single nucleotide polymorphism array. Plant Biot J 12:787-796

45. Watson IA, Luig NH (1966) Sr 15-a new gene for use in the classification of Puccinia graminis var. tritici. Euphytica 15:239-247

46. Wu J, Zeng Q, Wang Q, Liu S, Yu S, Mu J, Huang S, Sela H, Distelfeld A, Huang L (2018) SNP-based pool genotyping and haplotype analysis accelerate fine-mapping of the wheat genomic region containing stripe rust resistance gene Yr26. Theor Appl Genet 131:1481-1496

\section{Figures}

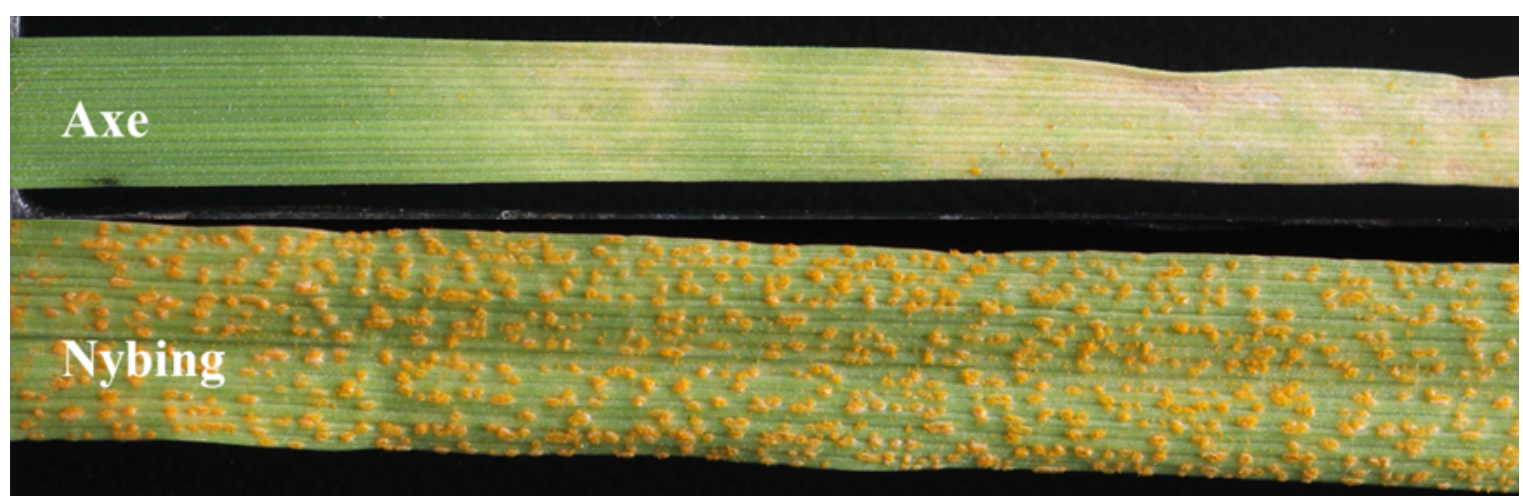

\section{Figure 1}

Infection types produced by parents Axe and Nyb against Pst pathotype134E16 A+Yr17+Yr27+ at 4th leaf stage 


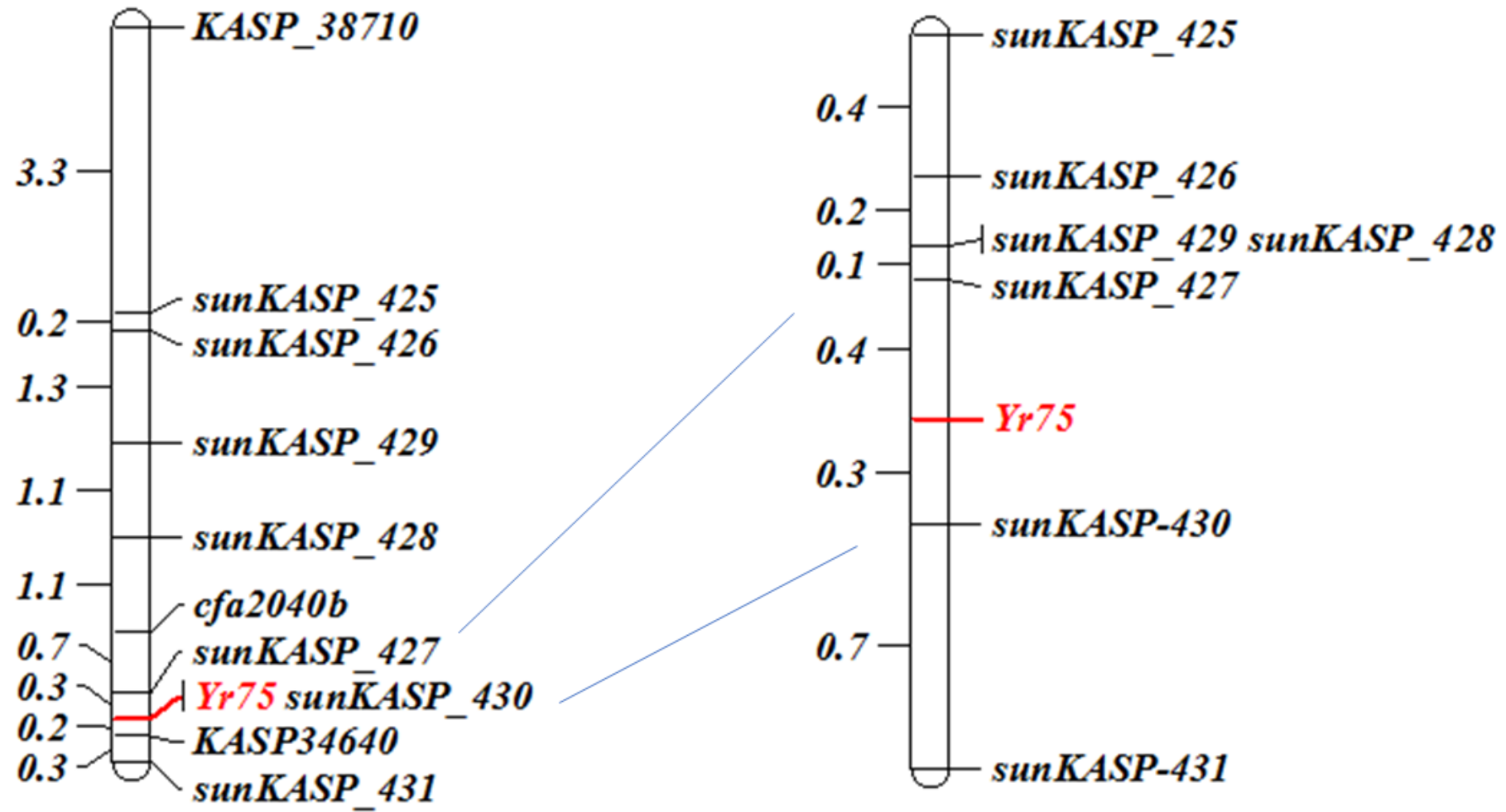

Figure 2

Low-resolution map of chromosome 7AL of Axe/Nyb RIL population (a) and high-resolution map of Axe/Nyb F2 population (b) 


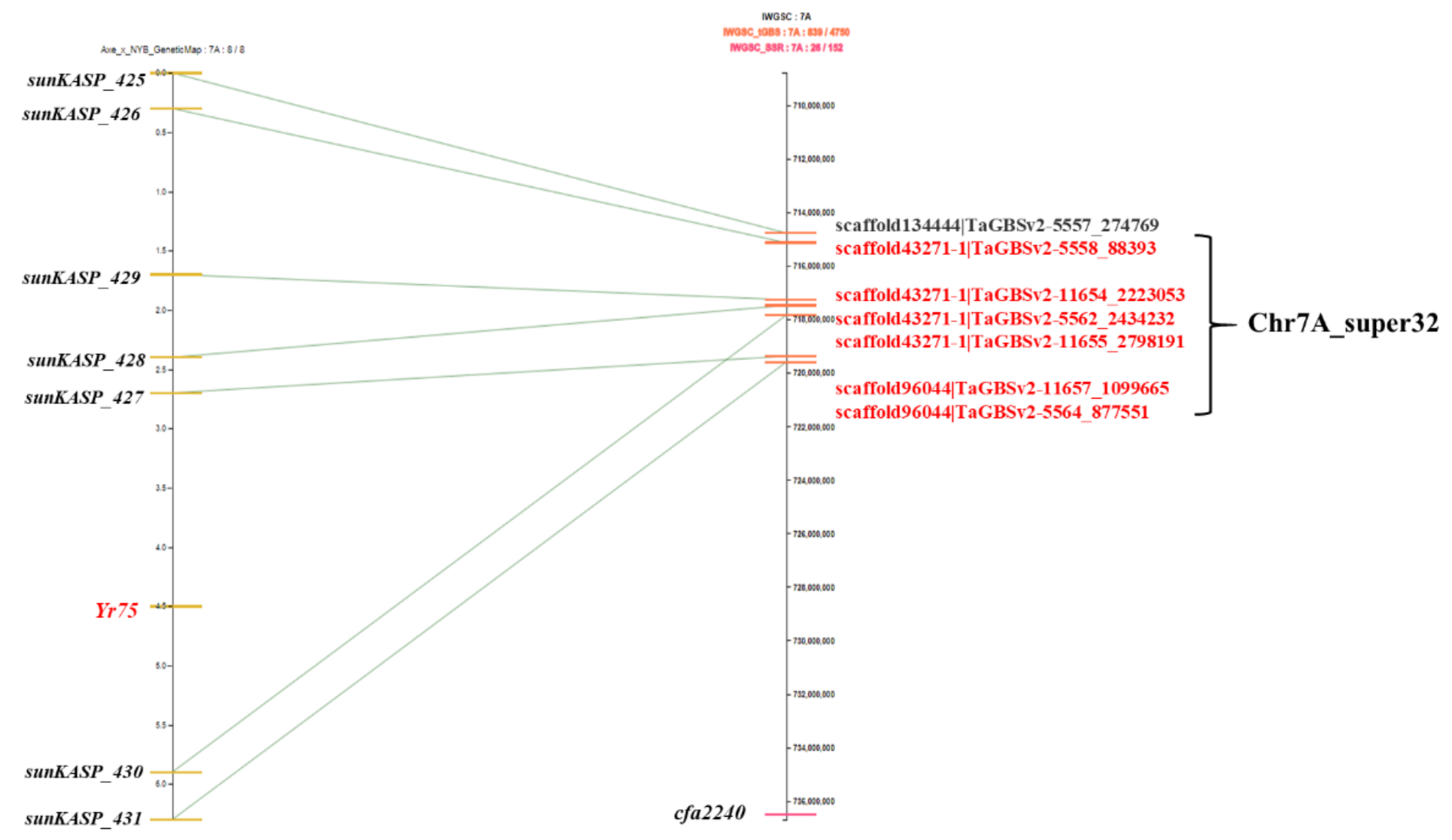

Figure 3

Position of Yr75 linked markers on chromosome 7AL in the IWGSC v1.0 Chinese Spring genome assembly. Figure constructed using Pretzel (Keeble-Gagnère et al. 2019) 